\title{
The Utilization of Crisp Code in Hybrid Reactor Studies
}

\author{
S. Anéfalos ${ }^{1}$, A. Deppman ${ }^{1}$, G. Silva ${ }^{1}$, J. R. Maiorino ${ }^{2}$, A. dos $\operatorname{Santos}^{2}$, S. B. Duarte ${ }^{3}$, O. A. P. Tavares ${ }^{3}$, and F. Garcia ${ }^{4}$ \\ 1) Instituto de Física - Universidade de São Paulo, \\ IFUSP, São Paulo, SP, Brazil \\ 2) Instituto de Pesquisas Energéticas e Nucleares - IPEN, \\ São Paulo, SP, Brazil \\ 3) Centro Brasileiro de Pesquisas Físicas, \\ $C B P F$, Rio de Janeiro, RJ, Brazil \\ 4) Universidade Estadual de Santa Cruz, Bahia, Brazil
}

Received on 1st August, 2005

\begin{abstract}
One of the main applications of the Hybrid Reactors (ADS - Accelerator Driven System) is the incineration of transuranics (TRU) by fast neutrons that emerge from a spallation source in a sub critical reactor waste burner $[1,2]$. For this application, an accurate description and prediction of spallation reaction is necessary, including all the characteristics concerning spatial and energetic angular distributions, spallation products and neutron multiplicity. To describe the nuclear reactions at intermediate and high energies, Monte Carlo calculations have been used. The CRISP package considers the intranuclear cascade (INC) that occurs during the spallation process in a realistic time-sequence approach in which all particles inside the nucleus can participate in the cascade and the nuclear density fluctuations are naturally taken into account during the process. The occupation number of each single particle level is considered as a function of time and a more realistic Pauli blocking mechanism can be performed. None of the existing models have effectively used all those features. The evaporation of protons and alpha particles are taken into account making possible the correct prediction of fissilities of actinides and pre-actinides [3]. Another implementation is the NN single-pion production reaction. This reaction is especially relevant if one is interested in neutron or proton multiplicities, since the creation/emission of pions is directly related with the excitation energy of the residual nucleus. We will present some results obtained with the CRISP package for proton-nucleus reaction at intermediate and high energies. This package was obtained by the coupling of the MCMC [4] and MCEF [5] codes, with the introduction of some improvements, such as better Pauli blocking mechanism, which constrains the residual nucleus energetic evolution to the Pauli Principle from the ground-state to the final compound-nucleus formed at the end of the intranuclear cascade process, and introduction of the most relevant resonant excitation and the NN single pion production channel. The results of interest for ADS development are consistent with the experimental data at different proton energies. More detailed calculations are being performed for studying other features of proton-nucleus reactions and with different targets.
\end{abstract}

\section{INTRODUCTION}

To overcome the need of final storage, incineration of transuranics, TRU, is being considered by using fast neutrons from a spallation source. It takes into account that the fission cross section is much higher than the capture cross section at these energies for most of TRU, and therefore transmuting long lived TRU in medium or short lived waste (fission fragments - FF). Besides the incineration of TRU and FF, a coupled system consisting of spallation source and a sub critical array of fuel, may have a positive gain of energy (net energy/energy to operate the accelerator). This system is known as ADS - Accelerator Driven System.

\section{A. Target}

One of the preferred options for an ADS target is the LeadBismuth Eutectic (LBE). Effects of spallation products on LBE corrosion control are one of the key problems to be investigated.

In most hybrid systems a thin window exists between the incident proton beam and the spallation source, needed to pro- tect the vacuum in the accelerator tube and being, at the same time, the first barrier to the spallation target. This window is submitted continuously to the proton irradiation. Among those problems are the radioactivity induced by the spallation products, changes in the chemical composition and embrittlement (Martensitic steels has $90 \%$ of Fe).

Some radioactive spallation products are:

${ }^{210} \mathrm{Po}\left(\mathrm{T}_{1 / 2}=138\right.$ days $)$ for Bismuth target,

${ }^{194} \mathrm{Hg}\left(\mathrm{T}_{1 / 2}=520 \mathrm{yr}\right)$ for Mercury and Lead targets,

${ }^{148} \mathrm{Gd}\left(\mathrm{T}_{1 / 2}=74.6 \mathrm{yr}\right)$ for Tungsten and Lead targets.

\section{THE CRISP PACKAGE}

The CRISP package here developed utilizes an algorithm that describes a many-body intranuclear cascade (INC) and evaporation/fission competition process, considering dynamic evaluation of the fermionic multicolisional process and the possibility of neutron, proton and alpha particle evaporation $[6,7]$. Fission process can also occur and competing with the evaporation process. The INC is considered in a realistic time- 
sequence approach and all particles inside the nucleus can participate in the cascade. The occupation number of each single particle level is considered as a function of time and a more realistic Pauli blocking mechanism was implemented.

\section{IMPLEMENTATIONS}

Another implementation is the NN direct single-pion production reactions. The channels that are present in the CRISP code are listed bellow:

$$
\begin{gathered}
p+p \rightarrow p+n+\pi^{+} \\
p+p \rightarrow p+p+\pi^{0} \\
n+p \rightarrow n n \pi^{+}+p p \pi^{-}
\end{gathered}
$$

The two-pions production occurs at about $1 \mathrm{GeV}$ and is being implemented.

The level density parameters present in CRISP are the same as described in [8]:

$\mathrm{a}_{n}{ }^{1 / 2}=\mathrm{a}^{1 / 2}(1-1.3 \Theta / \mathrm{A})$,

$\mathrm{a}_{p}{ }^{1 / 2}=\mathrm{a}^{1 / 2}(1+1.3 \Theta / \mathrm{A})$,

$\mathrm{a}_{\alpha}{ }^{1 / 2}=\mathrm{a}^{1 / 2}(1-3 / 2 \mathrm{~A})$

$\mathrm{a}_{f}=\mathrm{a}_{n} \times \mathrm{r}_{f}$,

where

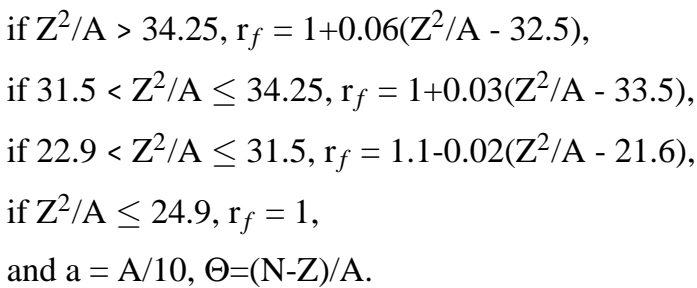

\section{RESULTS}

In Table 1, neutron multiplicities for proton induced reactions on $\mathrm{Pb}$ at different intervals of energy are given. Our model predictions agree very well with the cascade neutrons $(E \geq 20$ $\mathrm{MeV})$ and the evaporation neutrons $(2-20 \mathrm{MeV})$ [9]. Also in
Table 1 the CRISP results are compared with those obtained with other models.

During the irradiation a large amount of neutrons is generated. However, the process generates spallation products from the target material, in direct proportion to the energy of the proton beam. Their radiotoxicity is likely to exceed that of the fission products in the short term, which is relevant to operation and storage rather than final disposal. Ultimately the burning of actinides means that overall radiotoxicity is reduced greatly by 1000 years, and is less than that of the equivalent uranium ore.

Considering this, an important result is to calculate the spallation products that are generated in the target during the reactor operation. In figure 1 the spallation products resulting from a Lead target irradiated by $1 \mathrm{GeV}$ protons are shown. Although the results are preliminary, the calculated data agree very well with the experimental one. Analyzing this results one can estimate how much ${ }^{194} \mathrm{Hg}$, for example, could be generated.

In figure 2 the spallation products resulting from a Gold target irradiated by $0.8 \mathrm{GeV}$ protons are shown. Although the results are also preliminary, the calculated data agree very well with the experimental one.

In both figures one can note that there are some discrepancies in the curves with higher atomic numbers and higher atomic masses. This kind of effect is related to the initial interaction and we are working on it.

\section{CONCLUSIONS}

The particle multiplicities calculated with CRISP code agree with the experimental data, giving results as good as, or even better than other INC/Evaporation codes. The spallation products parabolas show a good agreement with experimental data, indicating that the INC and Evaporation/fission processes are being correctly simulated. The CRISP package can be used in ADS target design contributing with the correct prediction of important quantities.

\section{ACKNOWLEDGMENT}

We are thankful to FAPESP, CNPq and CAPES for their support.
[1] C. Rubbia and J. A. Rubio (CERN/LHC/96-11(EET)).

[2] C. Rubbia et al, (CERN/AT/95-44(ET)).

[3] A. Deppman et al., Phys. Rev Lett. 87, 182701 (2001).

[4] M. Gonçalves et al., Phys. Lett. B 406, 1 (1997).

[5] A. Deppman et al., Comp. Phys. Comm. 145, 385 (2002).

[6] Dostrovsky, I. et al. Phys. Rev. 111, no 6 (1958).
[7] A. Deppman et al., Comp. Phys. Comm. 145, 385 (2002)

[8] S. B. Duarte et al., Phys. Rev. Lett. 49, 536 (1982).

[9] A. Boudard et al., Phys. Rev. C 66, 044615 (2002).

[10] T. Enqvist et al., Nucl. Phys. A 686, 481 (2001).

[11] F. Rejmund et al., Nucl. Phys. A 683, 540 (2001). 
TABLE I: Neutron multiplicities in proton-induced reaction on $\mathrm{Pb}$ nuclei (second column) compared with the predictions of our model (third column) and those from other models [9].

\begin{tabular}{|c|c|c|c|c|c|c|c|}
\hline $\begin{array}{l}\text { Neutron } \\
\text { energy }\end{array}$ & Expt. & CRISP & $\begin{array}{l}\text { INCL4 } \\
\text { KHSv3p }\end{array}$ & $\begin{array}{l}\text { TIERCE } \\
\text { Cugnon }\end{array}$ & $\begin{array}{l}\text { LAHET } \\
\text { Bertini }\end{array}$ & $\begin{array}{l}\text { LAHET } \\
\text { ISABEL }\end{array}$ & $\begin{array}{l}\text { LAHET } \\
\text { Bertini-preq }\end{array}$ \\
\hline & \multicolumn{7}{|c|}{$P b \mathbf{E}_{p}=800 \mathrm{MeV}$} \\
\hline 0 - $2 \mathrm{MeV}$ & & 2.9 & 3.3 & $4.9^{P}$ & 5.61 & 5.13 & 5.37 \\
\hline 2-20 MeV & 6.5 & 6.6 & 6.8 & 6.9 & 8.63 & 6.63 & 7.12 \\
\hline$>20 \mathrm{MeV}$ & 1.9 & 2.2 & 2.5 & 2.2 & 1.75 & 1.92 & 2.13 \\
\hline \multirow[t]{2}{*}{ Total } & & 11.7 & 12.5 & 14.0 & 16.0 & 13.7 & 14.04 \\
\hline & \multicolumn{7}{|c|}{$P b \mathbf{E}_{p}=1200 \mathrm{MeV}$} \\
\hline о - $2 \mathrm{MeV}$ & & 3.3 & 3.4 & 5.8 & 6.35 & & 6.02 \\
\hline 2-20 MeV & 8.3 & 7.6 & 8.1 & 8.9 & 11.44 & & 9.86 \\
\hline > $20 \mathrm{MeV}$ & 2.7 & 2.6 & 3.1 & 2.8 & 2.45 & & 2.83 \\
\hline Total & & 13.5 & 14.7 & 17.4 & 20.2 & & 18.7 \\
\hline
\end{tabular}

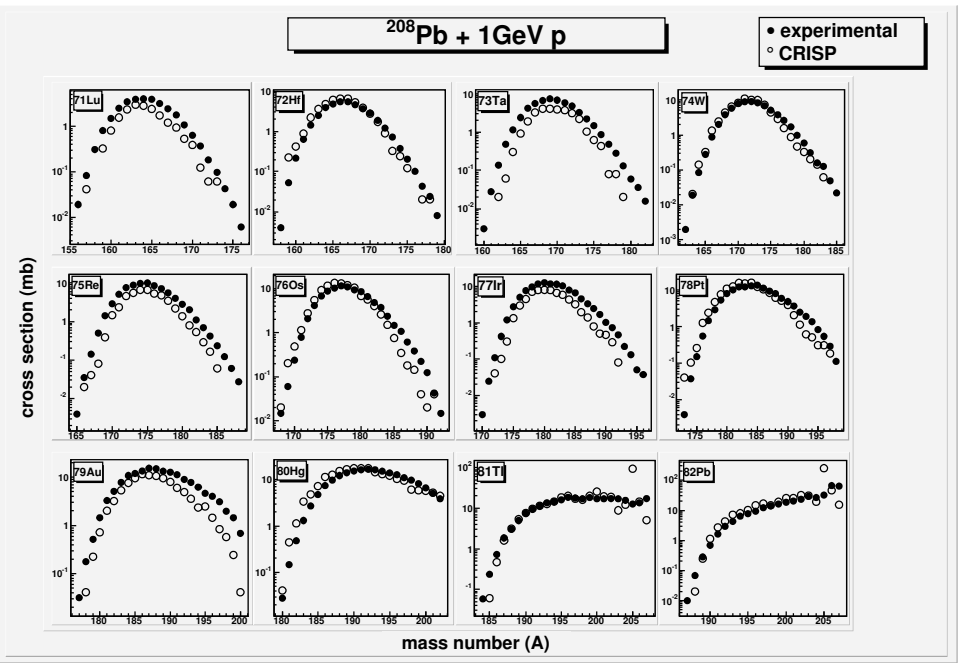

FIG. 1: Spallation products from a Lead target irradiated by $1 \mathrm{GeV}$ protons. The full circles are experimental data taken from [10]. The open circles are from CRISP calculations.

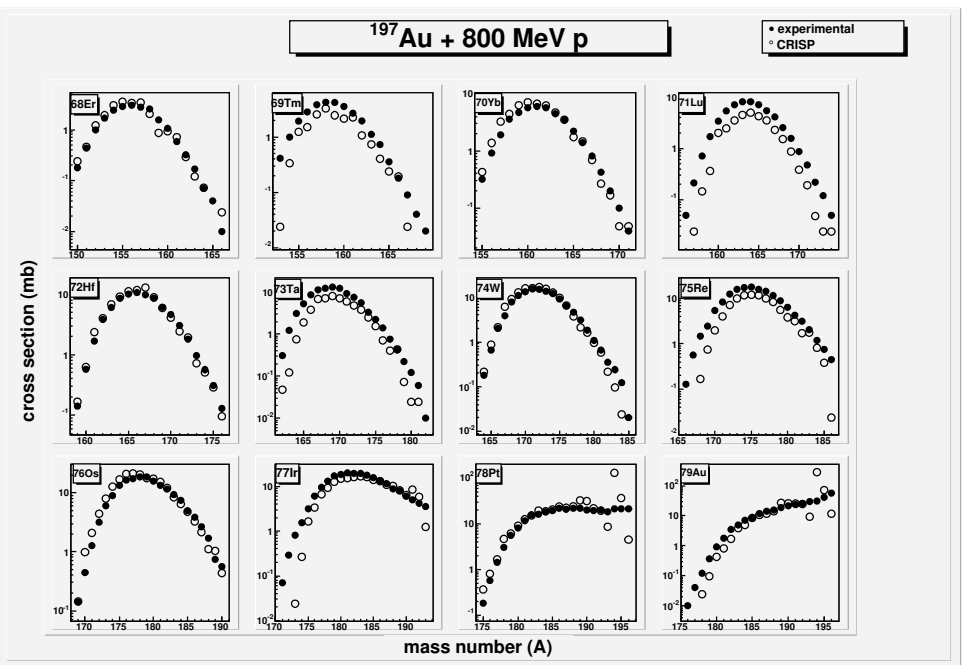

FIG. 2: Spallation products from a lead target irradiated by $0.8 \mathrm{GeV}$ protons. The full circles are experimental data taken from [11]. The open circles are from CRISP calculations. 